\title{
Nonlocality without inequalities *
}

\author{
GianCarlo Ghirardi ${ }^{\dagger}$ \\ Department of Theoretical Physics of the University of Trieste, \\ Istituto Nazionale di Fisica Nucleare, Sezione di Trieste, and \\ International Centre for Theoretical Physics "Abdus Salam", Trieste, Italy, \\ Luca Marinatto $0^{\ddagger}$ \\ Department of Theoretical Physics of the University of Trieste, and \\ Istituto Nazionale di Fisica Nucleare, Sezione di Trieste, Italy,
}

\begin{abstract}
We prove that every conceivable hidden variable model reproducing the quantum mechanical predictions of almost any entangled state must necessarily violate Bell's locality condition. The proof does not involve the consideration of any Bell inequality but it rests on simple set theoretic arguments and it works for almost any noncompletely factorizable state vector associated to any number of particles whose Hilbert spaces have arbitrary dimensionality.
\end{abstract}

PACS numbers: 03.65.-w, 03.65.Ud

\section{Introduction}

It is well known that the natural processes cannot be described by resorting to local realistic theories without conflicting with the experimentally verified predictions that quantum mechanics attaches to measurement outcomes. The proof of this fact is usually obtained by considering experiments which imply a violation of Bell's inequalities [1, 2, which are constraints on particular combinations of correlation functions that every local theory requires to be satisfied. Accordingly, experimental evidence of nonlocality is obtained by performing different correlation measurements onto sets of particles all prepared in identical entangled states [3]. In the literature there also exist proofs of nonlocality which do not make use of inequalities at all but simply rest on a clever use of EPR-like arguments [4] and algebraic manipulations of suitable chosen sets of operators. The first of these "nonlocality without inequalities" proofs is due to D. M. Greenberger, M. Horne and A. Zeilinger [5], but the most striking one has been exhibited by L. Hardy [6] who was able to rule out the existence of any local realistic theory accounting for the predictions of any entangled, but not maximally entangled, state of two spin- $1 / 2$ particles. Subsequent refinements and extensions of Hardy's proof which, due to its simplicity and generality, has been defined "the best version of Bell's theorem" [7, have been derived [8, 9, 10].

\footnotetext{
${ }^{*}$ Work supported in part by Istituto Nazionale di Fisica Nucleare, Sezione di Trieste, Italy

${ }^{\dagger}$ e-mail: ghirardi@ts.infn.it

${ }^{\ddagger}$ e-mail: marinatto@ts.infn.it
} 
The nice aspect of this approach derives from the fact that the experimental rejection of any local theory is simply obtained by verifying the occurrence of one particular joint measurement outcome [1]. The general theoretical proof of nonlocality we are going to exhibit represents a reformulation and a generalization of Hardy's argument valid for almost any non-completely factorizable state vector of a system composed of an arbitrary number of particles whose Hilbert spaces have arbitrary dimensionality. The quite general framework we will deal with is that of any conceivable theory completing quantum mechanics such that: (i) the most accurate specification it allows of the state of the physical system uniquely identifies the probabilities of all single and joint measurements outcomes, (ii) such probabilities, when appropriately averaged over, agree with the quantum mechanical ones, and (iii) it satisfies the mathematically precise Bell locality request [12] when the particles are space-like separated. On the contrary of Hardy's approach, our proof deals with simple set theoretic arguments avoiding EPR-counterfactual statements and makes use of the Bell locality condition rather than appealing to the Einstein locality condition ${ }^{1}$. From the experimental point of view, the procedure allows to identify the precise measurements which can yield, with a non-zero probability, outcomes putting into evidence the unavoidable "nonlocality" of every conceivable completion of quantum mechanics. Therefore, contrary to all nonlocality tests based on a violation of some Bell's inequality which require different correlation experiments, the tests based on a Hardy-like approach require simply the occurrence of a particular joint measurement outcome whose probability depends on the entangled state under consideration. The joint measurement, as we will see by exhibiting two equivalent nonlocality proofs, can involve, in general, either complicated multipartite measurements or multiple single-particle measurements. In the latter case, the advantage of dealing with simple observables is partially reduced by the fact that the probability of the desired outcome may be significantly reduced with respect to former case.

This paper is organized as follows: in the second section we make precise the notion of a local stochastic hidden variable model while in the third section we exhibit the proof of nonlocality without inequalities. Finally, in the fourth section an equivalent proof is given, having the advantage of dealing with single-particle observables.

\section{Local hidden variable models}

Before exhibiting the proof of nonlocality without inequalities, we identify the formal framework we will be dealing with throughout the paper and the precise locality request we will use. The framework consists of the so-called stochastic hidden variable theories for correlation experiments of the EPR-Bell type, experiments where observables are measured at spacelike separated locations. The idea underlying such theories is that the most complete specification of the state of an individual physical system is represented by the assignment of certain variables $\lambda$, which uniquely determine all (single or joint) probability distributions for possible outcomes to occur. More precisely, a stochastic hidden variable model for a correlation experiment, involving measurement processes performed on a $n$-partite quantum system described by the state

\footnotetext{
1 "But one supposition we should, in my opinion, absolutely hold fast: the real factual situation of the system $S_{2}$ is independent of what is done with the system $S_{1}$, which is spatially separated from the former". A. Einstein in Albert Einstein, Philosopher Scientist, Ed. by P.A.Schilp, p.85, Library of Living Philosophers, Evanston, Illinois (1949).
} 
$|\psi(1,2, \ldots, n)\rangle \in \mathcal{H}_{1} \otimes \cdots \otimes \mathcal{H}_{n}$ (where the dimensionality of $\mathcal{H}_{i}$ is arbitrary), consists of: (i) a set $\Lambda$ whose elements $\lambda$ are called hidden variables; (ii) a normalized and positive probability distribution $\rho$ defined on $\Lambda$; (iii) a set of probability distributions $P_{\lambda}\left(A_{i}=a, B_{j}=b, \ldots, Z_{k}=z\right)$ for the outcomes of single and joint measurements of any conceivable set of observables $\left\{A_{i}, B_{j}, \ldots, Z_{k}\right\}$ where each index $\{i, j, \ldots, k\}$ refers to a single particle or to a group of particles, such that:

$$
P_{\psi}\left(A_{i}=a, B_{j}=b, \ldots, Z_{k}=z\right)=\int_{\Lambda} d \lambda \rho(\lambda) P_{\lambda}\left(A_{i}=a, B_{j}=b, \ldots, Z_{k}=z\right) .
$$

The quantities at the left hand side of Eq. (2.11) are the probability distributions predicted by quantum mechanics concerning the outcomes $\{a, b, \ldots, z\}$ for the joint measurement of the observables $\left(A_{i}, B_{j} \ldots Z_{k}\right)$ respectively, when the system is associated the state vector $|\psi\rangle$. A deterministic hidden variable model is a particular instance of a stochastic model where all probabilities $P_{\lambda}$ can take only the values 0 or 1 .

Hidden variable models represent an extremely general class of theories completing quantum mechanics by means of the introduction of the parameters $\lambda$, which may be completely or only partially accessible, both practically or in principle, to the experimenter. For example the $\lambda_{\mathrm{s}}$ might simply reduce to the state vector $|\psi\rangle$ of the system, as happens in ordinary quantum mechanics, and in this case no average procedure over the distribution $\rho(\lambda)$ is necessary, or they might represent both the state vector $|\psi\rangle$ and the positions of all the particles of the system, as happens in Bohmian mechanics. In this last case, while $|\psi\rangle$ is considered as accessible, the positions are assumed to be in principle not accessible so that an average over their distribution is needed in order to reproduce the quantum predictions. In the most general case, the accessible knowledge about the actual physical situation can reduce simply to the knowledge of $\rho(\lambda)$ itself and the physical predictions are basically obtained by an averaging procedure. An example of such a situation is represented by Bell's model identifying the states of a spin- $1 / 2$ particle by unit vectors in a tridimensional Euclidean space [1].

Let us now make precise the locality condition which we impose to the theories we are interested in. When consideration is given to measurement processes taking place at spacelike separated regions, it is quite natural to require that all joint probability distributions satisfy the factorization property

$$
P_{\lambda}\left(A_{i}=a, B_{j}=b, \ldots, Z_{k}=z\right)=P_{\lambda}\left(A_{i}=a\right) P_{\lambda}\left(B_{j}=b\right) \ldots P_{\lambda}\left(Z_{k}=z\right) \quad \forall \lambda \in \Lambda .
$$

This factorizability request is commonly referred as Bell's locality condition [12], as opposed to the Einstein locality. In the particular case in which the most complete specification of the state of a physical system is given simply by the the state vector $|\psi\rangle$, i.e., within ordinary quantum mechanics, violation of the locality condition of Eq. (2.2) may be ascertained directly by considering appropriate observables. In fact, it is well-known that for any entangled state there exist joint probabilities which do not factorize. As a consequence, establishing that ordinary quantum mechanics is a genuine nonlocal theory is straightforward, while it is more difficult to prove unavoidable nonlocal structure of any conceivable completion of quantum mechanics. Finally, it is worth noticing that A. Fine [13] has proved that local stochastic hidden variable models are completely equivalent to local deterministic hidden variable models. As a consequence, all single and joint probability distributions $P_{\lambda}$ can be thought to assume values within the interval $[0,1]$ or, equivalently, the values 0 and 1 only. Nonetheless, in what follows we will keep on using the 
general notation $P_{\lambda}$, without stating explicitly whether we are dealing with a genuine stochastic hidden variable model or not.

\section{$3 \quad$ Nonlocality without inequalities for almost any entangled state}

Consider an $n$-partite quantum system described by the state vector $|\psi(1, \ldots, n)\rangle$ belonging to the Hilbert space $\mathcal{H}_{1} \otimes \cdots \otimes \mathcal{H}_{n}$, the dimension of each $\mathcal{H}_{i}, i=1, \ldots, n$ being greater or equal to 2 . Let us arbitrarily split the set of $n$ particles in two subsets, which we label as 1 and 2 in what follows, and suppose they involve particles located into two space-like separated regions. Finally, we consider the Schmidt decomposition of $|\psi\rangle$ in terms of appropriate orthonormal sets of states $\left\{\left|\alpha_{i}(1)\right\rangle\right\}$ and $\left\{\left|\beta_{i}(2)\right\rangle\right\}$, belonging to the Hilbert spaces of the first and the second group of particles respectively:

$$
|\psi(1,2)\rangle=\sum_{i} p_{i}\left|\alpha_{i}(1)\right\rangle \otimes\left|\beta_{i}(2)\right\rangle
$$

The weights $p_{i}$ are positive real numbers satisfying the normalization condition $\sum_{i} p_{i}^{2}=1$. Suppose now that the Schmidt decomposition of Eq.(3.1) involves at least two different weights, which we assume for simplicity to be the first two, so that $p_{1} \neq p_{2}$. Actually, this is the only hypothesis which is necessary for our proof (in fact, failure of this condition, as we will see, will not allow us to conclude anything concerning the existence or not of a local stochastic hidden variable model for the state $|\psi\rangle$ ), and it implies that we can prove nonlocality only for those non-completely factorizable states whose Schmidt coefficients are not all equal. Consequently, our proof does not apply, for example, to the maximally entangled states of $\mathbb{C}^{2} \otimes \mathbb{C}^{2}$ or to the $n$-partite GHZ states. To start with, let us define the following two $2 \times 2$ unitary matrices $U$ and $V$ whose entries depend on the weights $p_{1}$ and $p_{2}$ :

$U=\frac{1}{\sqrt{p_{1}+p_{2}}}\left[\begin{array}{cc}\sqrt{p_{2}} & -i \sqrt{p_{1}} \\ -i \sqrt{p_{1}} & \sqrt{p_{2}}\end{array}\right] \quad V=\frac{1}{\sqrt{p_{1}^{2}+p_{2}^{2}-p_{1} p_{2}}}\left[\begin{array}{cc}-i\left(p_{2}-p_{1}\right) & \sqrt{p_{1} p_{2}} \\ \sqrt{p_{1} p_{2}} & -i\left(p_{2}-p_{1}\right)\end{array}\right]$.

Now, define two orthonormal bases $\left\{\left|x_{+}(1)\right\rangle,\left|x_{-}(1)\right\rangle\right\}$ and $\left\{\left|y_{+}(1)\right\rangle,\left|y_{-}(1)\right\rangle\right\}$ belonging to the two-dimensional linear manifold of the first group of particles spanned by the vectors $\left\{\left|\alpha_{1}(1)\right\rangle,\left|\alpha_{2}(1)\right\rangle\right\}$, and two bases $\left\{\left|x_{+}(2)\right\rangle,\left|x_{-}(2)\right\rangle\right\}$ and $\left\{\left|y_{+}(2)\right\rangle,\left|y_{-}(2)\right\rangle\right\}$ for the two-dimensional linear manifold of the second group of particles spanned by the vectors $\left\{\left|\beta_{1}(2)\right\rangle,\left|\beta_{2}(2)\right\rangle\right\}$, according to:

$$
\begin{array}{ll}
{\left[\begin{array}{l}
\left|x_{+}(1)\right\rangle \\
\left|x_{-}(1)\right\rangle
\end{array}\right]=U\left[\begin{array}{l}
\left|\alpha_{1}(1)\right\rangle \\
\left|\alpha_{2}(1)\right\rangle
\end{array}\right]} & {\left[\begin{array}{l}
\left|y_{+}(1)\right\rangle \\
\left|y_{-}(1)\right\rangle
\end{array}\right]=V U\left[\begin{array}{l}
\left|\alpha_{1}(1)\right\rangle \\
\left|\alpha_{2}(1)\right\rangle
\end{array}\right]} \\
{\left[\begin{array}{l}
\left|x_{+}(2)\right\rangle \\
\left|x_{-}(2)\right\rangle
\end{array}\right]=U\left[\begin{array}{l}
\left|\beta_{1}(2)\right\rangle \\
\left|\beta_{2}(2)\right\rangle
\end{array}\right]} & {\left[\begin{array}{l}
\left|y_{+}(2)\right\rangle \\
\left|y_{-}(2)\right\rangle
\end{array}\right]=V U\left[\begin{array}{l}
\left|\beta_{1}(2)\right\rangle \\
\left|\beta_{2}(2)\right\rangle
\end{array}\right] .}
\end{array}
$$


The state $|\psi\rangle$ of Eq. (3.1) can be expressed in three equivalent forms by resorting to the basis vectors defined in Eqs. (3.3][3.4), as:

$$
\begin{aligned}
|\psi(1,2)\rangle & =i \sqrt{p_{1} p_{2}}\left[\left|x_{+}(1)\right\rangle\left|x_{-}(2)\right\rangle+\left|x_{-}(1)\right\rangle\left|x_{+}(2)\right\rangle\right]+\left(p_{2}-p_{1}\right)\left|x_{-}(1)\right\rangle\left|x_{-}(2)\right\rangle+\sum_{i>2} p_{i}\left|\alpha_{i}(1)\right\rangle\left|\beta_{i}(2)\right\rangle \\
& =i \sqrt{p_{1}^{2}+p_{2}^{2}-p_{1} p_{2}}\left|y_{-}(1)\right\rangle\left|x_{-}(2)\right\rangle+i \sqrt{p_{1} p_{2}}\left|x_{-}(1)\right\rangle\left|x_{+}(2)\right\rangle+\sum_{i>2} p_{i}\left|\alpha_{i}(1)\right\rangle\left|\beta_{i}(2)\right\rangle \\
& =i \sqrt{p_{1} p_{2}}\left[\left|x_{+}(1)\right\rangle\left|x_{-}(2)\right\rangle+i \sqrt{p_{1}^{2}+p_{2}^{2}-p_{1} p_{2}}\left|x_{-}(1)\right\rangle\left|y_{-}(2)\right\rangle+\sum_{i>2} p_{i}\left|\alpha_{i}(1)\right\rangle\left|\beta_{i}(2)\right\rangle\right.
\end{aligned}
$$

With the aim of exhibiting that particular set of joint probability distributions which cannot be described by any local hidden variable model, we consider the four operator $X_{1}, Y_{1}, X_{2}$ and $Y_{2}$. These are observables having as eigenstates associated to the eigenvalues +1 and -1 the orthonormal vectors $\left\{\left|x_{+}(1)\right\rangle,\left|x_{-}(1)\right\rangle\right\},\left\{\left|y_{+}(1)\right\rangle,\left|y_{-}(1)\right\rangle\right\},\left\{\left|x_{+}(2)\right\rangle,\left|x_{-}(2)\right\rangle\right\}$ and $\left\{\left|y_{+}(2)\right\rangle,\left|y_{-}(2)\right\rangle\right\}$ respectively, while they act as the null operator in the manifolds orthogonal to the bidimensional ones corresponding to the non-zero eigenvalues. According to Eq. (3.5) the quantum joint probabilities concerning the set of observables $X_{1}, Y_{1}, X_{2}$ and $Y_{2}$ satisfy the following relations:

$$
\begin{aligned}
P_{\psi}\left(X_{1}=+1, X_{2}=+1\right) & =0 \\
P_{\psi}\left(Y_{1}=+1, X_{2}=-1\right) & =0 \\
P_{\psi}\left(X_{1}=-1, Y_{2}=+1\right) & =0 \\
P_{\psi}\left(Y_{1}=+1, X_{2}=0\right) & =0 \\
P_{\psi}\left(X_{1}=0, Y_{2}=+1\right) & =0 \\
P_{\psi}\left(Y_{1}=+1, Y_{2}=+1\right) & =\frac{p_{1}^{2} p_{2}^{2}\left(p_{1}-p_{2}\right)^{2}}{\left(p_{1}^{2}+p_{2}^{2}-p_{1} p_{2}\right)^{2}} .
\end{aligned}
$$

Since we have supposed that the (strictly positive) weights $p_{1}$ and $p_{2}$ are such that $p_{1} \neq p_{2}$, the joint probability of Eq. (3.11) is different from zero and, as we will see, this is the crucial relation which will allow us to deny the existence of a local realistic description for the state under consideration. Suppose that a local stochastic hidden variable model reproducing, in accordance with Eq. (2.1), the quantum predictions for the state $|\psi\rangle$, exists. Accordingly, if we consider for example Eq. (3.6), we must have:

$$
\begin{aligned}
P_{\psi}\left(X_{1}=+1, X_{2}=+1\right) & =\int_{\Lambda} d \lambda \rho(\lambda) P_{\lambda}\left(X_{1}=+1, X_{2}=+1\right) \\
& =\int_{\Lambda} d \lambda \rho(\lambda) P_{\lambda}\left(X_{1}=+1\right) P_{\lambda}\left(X_{2}=+1\right)=0
\end{aligned}
$$

where the second equality is implied by the locality condition of Eq. (2.2). The last equality of Eq. (3.12) can be fulfilled if and only if the product $P_{\lambda}\left(X_{1}=+1\right) P_{\lambda}\left(X_{2}=+1\right)$ vanishes almost 
everywhere ${ }^{2}$ within $\Lambda$. An equivalent result holds for Eqs. (3.7.3.11), leading to:

$$
\begin{aligned}
& P_{\lambda}\left(X_{1}=+1\right) P_{\lambda}\left(X_{2}=+1\right)=0 \\
& P_{\lambda}\left(Y_{1}=+1\right) P_{\lambda}\left(X_{2}=-1\right)=0 \\
& P_{\lambda}\left(X_{1}=-1\right) P_{\lambda}\left(Y_{2}=+1\right)=0 \\
& P_{\lambda}\left(Y_{1}=+1\right) P_{\lambda}\left(X_{2}=0\right)=0 \\
& P_{\lambda}\left(X_{1}=0\right) P_{\lambda}\left(Y_{2}=+1\right)=0 \\
& P_{\lambda}\left(Y_{1}=+1\right) P_{\lambda}\left(Y_{2}=+1\right) \neq 0,
\end{aligned}
$$

where the first five equations are supposed to hold almost everywhere within $\Lambda$, while the sixth equation has to be satisfied in a subset of $\Lambda$ whose measure according to the distribution $\rho(\lambda)$ is non-zero. Before proceeding with the proof we note that, in the special case where the parameter $\lambda$ is the state vector $|\psi\rangle$, as happens in ordinary quantum mechanics, a violation of the previous constraints is immediately shown. In fact, given the particular state $|\psi\rangle$ of Eq. (3.5) both $P_{\lambda=\psi}\left(X_{1}=+1\right)$ and $P_{\lambda=\psi}\left(X_{2}=+1\right)$ are different from zero, thus contradicting Eq. (3.13).

In order to prove the more general result that no conceivable local stochastic hidden variable model can simultaneously satisfy the equations Eqs. (3.13) 3.18), a manipulations of those equations is requested. To this end, let us sum Eq. (3.14) and (3.16) so that, taking into account that $P_{\lambda}\left(X_{2}=-1\right)+P_{\lambda}\left(X_{2}=+0\right)+P_{\lambda}\left(X_{2}=+1\right)=1$, we obtain:

$$
P_{\lambda}\left(Y_{1}=+1\right)\left[1-P_{\lambda}\left(X_{2}=+1\right)\right]=0 .
$$

Similarly, summing Eqs. (3.15) and (3.17) we have:

$$
\left[1-P_{\lambda}\left(X_{1}=+1\right)\right] P_{\lambda}\left(Y_{2}=+1\right)=0 .
$$

Now let us partition the set of hidden variables $\Lambda$ and define the following subsets $A, B$ and $C$ as:

$$
\begin{aligned}
& A=\left\{\lambda \in \Lambda \mid P_{\lambda}\left(X_{1}=+1\right)=0\right\} \\
& B=\left\{\lambda \in \Lambda \mid P_{\lambda}\left(X_{2}=+1\right)=0\right\} \\
& C=\Lambda-(A \cup B) .
\end{aligned}
$$

Since $\Lambda-(A \cup B)=(\Lambda-A) \cap(\Lambda-B)$, we have that, for all $\lambda$ belonging to $C, P_{\lambda}\left(X_{1}=\right.$ $+1) P_{\lambda}\left(X_{2}=+1\right) \neq 0$. If the set $C$ would have a non-zero measure according to the distribution $\rho$, i.e., if $\int_{C} d \lambda \rho(\lambda) \neq 0$, there would be a violation of Eq. (3.13) and, consequently, of Eq. (3.6). Therefore, in order to fulfill Eq. (3.13), the set $A \cup B$ must coincide with $\Lambda$ apart from a set of zero measure, and we are left only with hidden variables belonging to either $A$ or $B$. If $\lambda$ belongs to $A$ then, by definition, $P_{\lambda}\left(X_{1}=+1\right)=0$, so that Eq. (3.20) can be satisfied only if $P_{\lambda}\left(Y_{2}=+1\right)=0$. Equivalently, if $\lambda$ belongs to $B$ then $P_{\lambda}\left(X_{2}=+1\right)=0$ and, according to Eq. (3.19), $P_{\lambda}\left(Y_{1}=+1\right)=0$. Hence, for any $\lambda \in A \cup B$ either $P_{\lambda}\left(Y_{1}=+1\right)=0$ or $P_{\lambda}\left(Y_{2}=+1\right)=0$, a fact leading to a contradiction of Eq. (3.18), which requires that there is a set of nonzero $\rho$-measure within $\Lambda$ where both probabilities do not vanish.

\footnotetext{
${ }^{2}$ By the expression "almost everywhere" it is meant that the argument of the integral may be different from zero at most within a non-empty set $\Gamma$ such that $\int_{\Gamma} d \lambda \rho(\lambda)=0$.
} 
To summarize, the simple proof we have just presented shows that it is not possible to exhibit any stochastic hidden variable model, satisfying Bell's locality condition of Eq. (2.2), which can account for the quantum mechanical predictions of almost any $n$-partite quantum entangled state $|\psi\rangle \in \mathcal{H}_{1} \otimes \cdots \otimes \mathcal{H}_{n}$, whose Schmidt decomposition, for any splitting of the particles, contains at least two different weights.

Exactly like in the original Hardy's proof [6], the experimental test of nonlocality for the entangled states we are considering, simply consists in testing the occurrence of the joint measurement outcomes of Eq. (3.11) whose probability

$$
P_{\psi}\left(Y_{1}=+1, Y_{2}=+1\right)=\frac{p_{1}^{2} p_{2}^{2}\left(p_{1}-p_{2}\right)^{2}}{\left(p_{1}^{2}+p_{2}^{2}-p_{1} p_{2}\right)^{2}}
$$

does not vanish whenever $p_{1}, p_{2} \neq 0$ and $p_{1} \neq p_{2}$.

\section{An equivalent proof}

To experimentally test the probability of Eq. (3.24), one has to perform measurements of the multipartite observables $Y_{1}$ and $Y_{2}$. Since they might involve (possibly large) groups of particles, an experiment like this could be quite complicated to perform from a practical point of view. In order to overcome this problem, we are going to exhibit now a modification of the previous proof which makes use of repeated application of the Schmidt decomposition of the $n$-partite state in order to identify a non-zero joint probability distribution conflicting with Bell's locality, which involves only single-particle observables. The only drawback of this equivalent proof consists, as we will see, in a possibly reduced value for the probability of the outcome conflicting with locality condition, which in general becomes smaller when the number $n$ of particles increases.

We start by considering a tripartite system and later we will show how to generalize the proof to cover the case of an arbitrary number of particles. Consider the Schmidt decomposition of a tripartite state $|\psi(1,2,3)\rangle$ in terms of a set of bipartite orthonormal states of the first and the second particle $\left\{\left|\phi_{k}(1,2)\right\rangle\right\}$, and of a set of orthonormal states of the third particle $\left\{\left|\tau_{k}(3)\right\rangle\right\}$ :

$$
|\psi(1,2,3)\rangle=\sum_{k} q_{k}\left|\phi_{k}(1,2)\right\rangle \otimes\left|\tau_{k}(3)\right\rangle
$$

where $q_{k} \geq 0$ and $\sum_{k} q_{k}^{2}=1$. Suppose now that, within the orthonormal set $\left\{\left|\phi_{k}(1,2)\right\rangle\right\}$, there exists a state, whose associated $q_{k}$ is different from zero, let us say $\left|\phi_{1}(1,2)\right\rangle$, such that at least two different weights appear in its Schmidt decomposition ${ }^{3}$. By performing the unitary transformations $U$ and $V$ as defined in Eqs. (3.3) 3.4), the state $|\psi(1,2,3)\rangle$ of Eq. (4.1) can be decomposed in the following form:

$$
\begin{aligned}
|\psi(1,2,3)\rangle= & q_{1}\left(i \sqrt{p_{1} p_{2}}\left[\left|x_{+}(1)\right\rangle\left|x_{-}(2)\right\rangle+\left|x_{-}(1)\right\rangle\left|x_{+}(2)\right\rangle\right]+\left(p_{2}-p_{1}\right)\left|x_{-}(1)\right\rangle\left|x_{-}(2)\right\rangle\right) \otimes\left|\tau_{1}(3)\right\rangle \\
& +q_{1}\left(\sum_{i>2} p_{i}\left|\alpha_{i}(1)\right\rangle\left|\beta_{i}(2)\right\rangle\right) \otimes\left|\tau_{1}(3)\right\rangle+\sum_{k>1} q_{k}\left|\phi_{k}(1,2)\right\rangle \otimes\left|\tau_{k}(3)\right\rangle
\end{aligned}
$$

Moreover, two other decompositions exist in analogy with Eq. (3.5).

\footnotetext{
${ }^{3}$ Actually the tripartite states for which our proof holds, are the ones whose Schmidt decomposition, performed by grouping the particles in every possible manner, contains at least one bipartite entangled state for an arbitrary pair of particles which satisfies the above request.
} 
Denoting by $T_{3}$ the single-particle observable of the Hilbert space of the third particle having the vectors $\left\{\left|\tau_{k}(3)\right\rangle\right\}$ as its eigenstates associated to a set of eigenvalues $\left\{t_{k}\right\}$ which we may choose so that $t_{1}$ is a non-degenerate eigenvalue, the following quantum probability distributions hold for the state of Eq. (4.2):

$$
\begin{aligned}
P_{\psi}\left(X_{1}=+1, X_{2}=+1, T_{3}=t_{1}\right) & =0 \\
P_{\psi}\left(Y_{1}=+1, X_{2}=-1, T_{3}=t_{1}\right) & =0 \\
P_{\psi}\left(X_{1}=-1, Y_{2}=+1, T_{3}=t_{1}\right) & =0 \\
P_{\psi}\left(Y_{1}=+1, X_{2}=0, T_{3}=t_{1}\right) & =0 \\
P_{\psi}\left(X_{1}=0, Y_{2}=+1, T_{3}=t_{1}\right) & =0 \\
P_{\psi}\left(Y_{1}=+1, Y_{2}=+1, T_{3}=t_{1}\right) & \neq 0 .
\end{aligned}
$$

Once again the existence of a local and stochastic hidden variable model reproducing the quantum mechanical probability distributions of Eqs. (4.3 4.8), implies the following relations:

$$
\begin{aligned}
P_{\lambda}\left(X_{1}=+1\right) P_{\lambda}\left(X_{2}=+1\right) P_{\lambda}\left(T_{3}=t_{1}\right) & =0 \\
P_{\lambda}\left(Y_{1}=+1\right) P_{\lambda}\left(X_{2}=-1\right) P_{\lambda}\left(T_{3}=t_{1}\right) & =0 \\
P_{\lambda}\left(X_{1}=-1\right) P_{\lambda}\left(Y_{2}=+1\right) P_{\lambda}\left(T_{3}=t_{1}\right) & =0 \\
P_{\lambda}\left(Y_{1}=+1\right) P_{\lambda}\left(X_{2}=0\right) P_{\lambda}\left(T_{3}=t_{1}\right) & =0 \\
P_{\lambda}\left(X_{1}=0\right) P_{\lambda}\left(Y_{2}=+1\right) P_{\lambda}\left(T_{3}=t_{1}\right) & =0 \\
P_{\lambda}\left(Y_{1}=+1\right) P_{\lambda}\left(Y_{2}=+1\right) P_{\lambda}\left(T_{3}=t_{1}\right) & \neq 0 .
\end{aligned}
$$

As before, the first five equations must be satisfied almost everywhere within $\Lambda$, while the last one has to be satisfied in a set of non-zero measure with respect to the distribution $\rho(\lambda)$.

Let us now show that a local stochastic hidden variable model satisfying Eqs. (4.9.4.14) cannot exist. The procedure we are going to follow consists in splitting the set of hidden variables $\Lambda$ into two complementary and disjoint subsets $\Omega_{1}$ and $\Omega_{2}$, referring to the possible values of the probability distribution for the outcome $t_{1}$ of the observable $T_{3}$. They are defined as $\Omega_{1}=\left\{\lambda \in \Lambda \mid P_{\lambda}\left(T_{3}=t_{1}\right)=0\right\}$ and $\Omega_{2}=\left\{\lambda \in \Lambda \mid P_{\lambda}\left(T_{3}=t_{1}\right) \neq 0\right\}$. Given any value of the hidden variable $\lambda$, two possible cases can occur: either $\lambda \in \Omega_{1}$ or $\lambda \in \Omega_{2}$. If $\lambda \in \Omega_{1}$, the left hand side of Eq. (4.14) vanishes and the equation cannot be satisfied. If $\lambda$ belongs to $\Omega_{2}$ where $P_{\lambda}\left(T_{3}=t_{1}\right) \neq 0$, the equations from (4.9) to (4.14) reduce to Eqs (3.13 3.18) respectively. This being the case, we can apply the previous arguments to conclude that no local stochastic hidden variable model exists which can reproduce the quantum probabilities for all tripartite states, whose Schmidt decomposition involves at least one bipartite state having at least two different weights in its decomposition. As before, this approach uniquely determines the set of outcomes of those joint measurements whose occurrence constitutes the experimental proof of nonlocality. Contrary to the first method those measurements involve only elementary singleparticle measurements, which are simpler to perform, but the probability of such an event:

$$
P_{\psi}\left(Y_{1}=+1, Y_{2}=+1, T_{3}=t_{1}\right)=q_{1}^{2} \cdot \frac{p_{1}^{2} p_{2}^{2}\left(p_{1}-p_{2}\right)^{2}}{\left(p_{1}^{2}+p_{2}^{2}-p_{1} p_{2}\right)^{2}}
$$

is smaller or equal to that of Eq. (3.24). 
The argument can be generalized in a straightforward way to any number of particles. In fact, given a four-particle state, we first perform its Schmidt decomposition in terms of orthonormal tripartite states $\left\{\left|\phi_{k}(1,2,3)\right\rangle\right\}$ and single-particle states $\left\{\left|\sigma_{k}(4)\right\rangle\right\}$ and then we check whether at least one tripartite state of the decomposition belongs to the set of states which we have just proven not to admit a local hidden variable description. If this is the case we define two disjoint and complementary sets of $\Lambda$, that is the sets $\Theta_{1}=\left\{\lambda \in \Lambda \mid P_{\lambda}\left(S_{4}=s_{1}\right)=0\right\}$ and $\Theta_{2}=$ $\left\{\lambda \in \Lambda \mid P_{\lambda}\left(S_{4}=s_{1}\right) \neq 0\right\}$, where $S_{4}$ is an observable of the Hilbert space of the fourth particle whose eigenstates are the vectors $\left\{\left|\sigma_{i}(4)\right\rangle\right\}$ and for which $s_{1}$ is not a degenerate eigenvalue. We then get quantum probability distributions of the form of Eqs. (4.9]4.14), where at the left hand side the extra multiplicative factor $P_{\lambda}\left(S_{4}=s_{1}\right)$ appears. Once again, if $\lambda \in \Theta_{1}$ the analogous expression to Eq. (4.14) containing the extra factor cannot be satisfied, while, when $\lambda \in \Theta_{2}$, we get back to the previous case. This proves that, step by step, we can generalize our argument to any number of particles. Note that the $n$-particle entangled states for which our proof holds are those for which, by considering all conceivable Schmidt decompositions in terms of bipartite states and $(n-2)$ single-particle states, at least one entangled bipartite state involving at least two different weights in its Schmidt decomposition appears. Such a set contains almost all entangled states of $n$-particles.

\section{Conclusions}

In this paper we have proven that every conceivable (deterministic or stochastic) hidden variable model reproducing the quantum mechanical predictions of almost any entangled state must necessarily violate Bell's locality condition. Two proofs of nonlocality have been exhibited, both of them not involving the consideration of any Bell inequality but simply resting on straightforward set theoretic arguments.

\section{References}

[1] J.S. Bell, Physics 1, 195 (1964).

[2] J.F. Clauser, M.A. Horne, A. Shimony, and R.A. Holt, Phys. Rev. Lett. 23, 880 (1969).

[3] A. Aspect, P. Grangier, and G. Roger, Phys. Rev. Lett. 47, 460 (1981).

[4] A. Einstein, N. Rosen and B. Podolsky, Phys. Rev. 47, 777 (1935).

[5] D.M. Greenberger, M. Horne, and A. Zeilinger in Bell's Theorem, Quantum Theory, and Conceptions of the Universe, M. Kafatos ed., Kluwer, Dordrecht (1989).

[6] L. Hardy, Phys. Rev. Lett. 71, 1665 (1993).

[7] N.D. Mermin, Ann. N.Y. Acad. Sci. 755, 616 (1995).

[8] Sheldon Goldstein, Phys. Rev. Lett. 721951 (1994).

[9] L. Hardy, in New Developments on Fundamental Problems in Quantum Physics, edited by M. Ferrero and A. van der Merwe, Kluwer, Dordrecht, 163 (1997). 
[10] D. Boschi, S. Branca, F. De Martini, and L. Hardy, Phys. Rev. Lett. 79, 2755 (1997).

[11] G. Di Giuseppe, F. De Martini, and D. Boschi, Phys. Rev. A 56, 176 (1997).

[12] J.S. Bell, Bertlemann's socks and the nature of reality in John S. Bell on The Foundations of Quantum Mechanics, M. Bell, K. Gottfried \& M. Veltman eds., World Scientific (2001).

[13] A. Fine, Phys. Rev. Lett. 48, 291 (1982). 